\title{
Pulse-based internal calibration of polarimetric SAR
}

\author{
Dall, Jørgen; Skou, Niels; Christensen, Erik Lintz
}

Published in:

Proceedings of the International Geoscience and Remote Sensing Symposium

Link to article, DOI:

10.1109/IGARSS.1994.399693

Publication date:

1994

Document Version

Publisher's PDF, also known as Version of record

Link back to DTU Orbit

\section{Citation $(A P A)$ :}

Dall, J., Skou, N., \& Christensen, E. L. (1994). Pulse-based internal calibration of polarimetric SAR. In Proceedings of the International Geoscience and Remote Sensing Symposium: Surface and Atmospheric Remote Sensing: Technologies, Data Analysis and Interpretation (pp. 2205-2208). IEEE. https://doi.org/10.1109/IGARSS.1994.399693

\section{General rights}

Copyright and moral rights for the publications made accessible in the public portal are retained by the authors and/or other copyright owners and it is a condition of accessing publications that users recognise and abide by the legal requirements associated with these rights.

- Users may download and print one copy of any publication from the public portal for the purpose of private study or research.

- You may not further distribute the material or use it for any profit-making activity or commercial gain

- You may freely distribute the URL identifying the publication in the public portal 


\title{
Pulse-Based Internal Calibration of Polarimetric SAR
}

\author{
Jørgen Dall, Niels Skou, Erik Lintz Christensen
}

Electromagnetics Institute, Technical University of Denmark,
DK-2800 Lyngby, Denmark

Phone: +4542881444 , Fax: +4545931634

\begin{abstract}
Internal calibration greatly diminishes the dependence on calibration target deployment compared to external calibration. Therefore Electromagnetics Institute (EMI) at the Technical University of Denmark (TUD) has equipped its polarimetric SAR, EMISAR, with several calibration loops and developed a procedure calibrating the entire system except a few passive components which must be measured in the laboratory. The calibration procedure has a great potential as it takes into account the SAR point target response (PTR) unlike most other internal calibration schemes. However, it requires a highly stable system.
\end{abstract}

\section{INTRODUCTION}

Internal calibration is often implemented by injecting a calibration tone during mapping. This approach has two disadvantages. Firstly, the system is only calibrated at a single frequency located outside the pulse spectrum. Secondly, major parts of the system cannot be included by this procedure. Using calibration pulses with the same encoding as used for mapping, the system can be amplitude and phase calibrated over the full spectrum. Furthermore, the built-in calibration paths allow the pulse-based procedure to calibrate almost the entire system, including both the transmitter and the receiver, but not the antenna which must be measured in an anechoic chamber. One drawback is that the internal calibration cannot take place simultaneously with the mapping, and consequently the system must be so stable that pre-map and post-map calibration pulses are adequate.

The cross-talk reduction that can be achieved with external calibration procedures is limited by the lact that the co- and crosspolarised antenna patterns are not necessarily proportional. Such limitations do not exist in internal calibration based on in-flight calibration pulses and laboratory measurements of a few components.

The internal calibration procedure developed for EMISAR (Christensen, 1994) comprises channel amplitude- and phase-imbalance correction, absolute radiometric calibration, and cross-talk elimination. Although not addressed in this paper, range calibration, STC calibration, and noise estimation is undertaken as well.

The calibration procedure provides the complex scattering matrix, $S$, which is a $2 \times 2$ matrix, $\left(S_{i j}\right)$, where $j \in\{H, V\}$ is the transmit polarisation and $\mathrm{i} \in(\mathrm{H}, \mathrm{V})$ is the receive polarisation. After the absolute radiometric calibration the energy of a point target (integration in range and azimuth) equals the radar cross section divided by $4 \pi$. Using the energy rather than the peak intensity implies that for a homogeneous distributed target the ensemble average of the pixel intensity equals the radar brightness divided by $4 \pi$ (Dall, 1994). In turn, the radar brightness is defined as sigma nought divided by sine to the local incidence angle.

The above definition of radiometric calibration is related to the well-known integral method (Gray, 1990). A one-dimensional version of this method is used to extract amplitude information from the compressed calibration pulses. The "calibration integral", $\hat{\mathbf{P}}$, corresponding to a quad-polarised calibration pulse, $\mathbf{P}$, is defined as a $2 \times 2$ matrix in which each of the complex elements has an amplitude equal to the square root of the pulse energy, and a phase equal to the pulse phase at the peak position or alternatively the phase of the complex pulse integral. In order to take the PTR into account it is crucial that the same calibration definition is applied to the internal calibration pulses as to the calibrated imagery.

Skou (1992) has previously presented the hardware aspects of the pulse-based internal calibration method. Therefore this paper concentrates on the usage of the calibration signals and laboratory

0-7803-1497-2/94 \$4.00 @ 1994 IEEE measurements for data calibration. Preliminary results are presented and compared with those obtained with external calibration techniques.

\section{BACKSCATTERED SIGNAL}

Fig. 1 outlines the block diagram of the polarimetric SAR. In the figure the five latching circulators constituting the polarisation switch are set up for $\mathrm{H}$ transmission. The transmitter and receiver switches are shown in the mapping positions (bold). With the switches in the opposite positions (shaded) the internal calibration loops are exercised. During mapping the shutter switches are transparent.

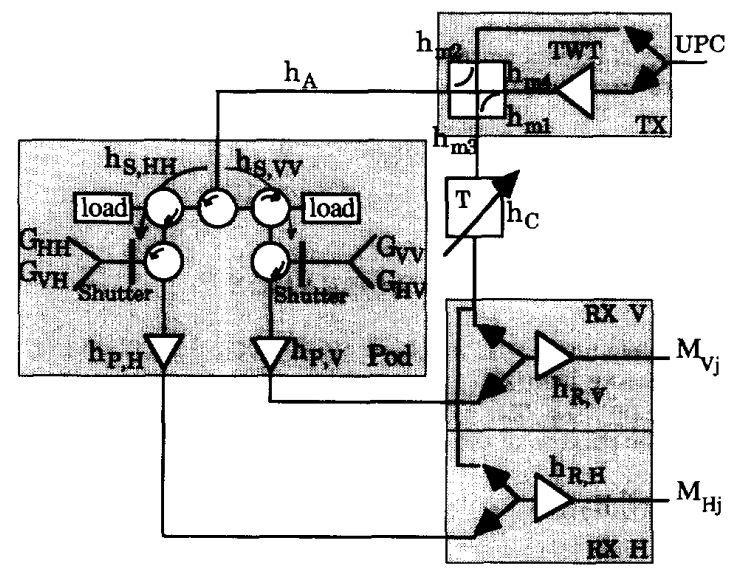

Fig. 1. SAR block diagram with calibration loops.

For simplicily, all components except the receivers, $h_{R, V}$ and $h_{R, H}$, are assumed to have a large bandwidth compared to that of the transmitted pulses. This implies that the impulse response (IPR), $h_{x}$. where $x$ is a generic index, is approximated by a complex constant multiplied by a $\delta$-function, $h_{x}=\delta(r) C_{x}$. Also for simplicity, several waveguide and coax connections are included in adjacent components in Fig. 1 .

Like the scattering process, the individual subsystems are expressed by $2 \times 2$ matrices, where the ( $i, j$ )-element is the $i$-channel IPR that is caused by a $j$-channel stimulus $(i, j \in\{H, V\})$. The measured signal is the convolution of these matrices

$$
\mathbf{M}=\mathbf{R} * \mathbf{A} * \mathbf{E}_{a} * \mathbf{S} * \mathbf{G} * \mathbf{C}_{S} * \mathbf{T} * \mathbf{E}_{r}
$$

$\mathbf{E}_{\mathrm{r}}$ describes the range encoded pulse just after the TWT. $\mathbf{T}$ is the transmitter matrix accounting for the transmission properties from the TWT to the polarisation switch. $\mathbf{C}_{S}$ accounts for the polarisation switch during transmission. The complex antenna gain is characterised by $\mathbf{G}$ and equivalently the effective area of the receiving antenna by $\mathbf{A} . \mathbf{S}$ is the scene scattering matrix. $\mathbf{E}_{\mathbf{a}}$ accounts for the azimuth encoding i.e. the Doppler history, range attenuation etc. Finally the receiver matrix $\mathbf{R}$ includes the transmission parameters from the receiver antenna port through the receivers.

* denotes matrix convolution which is identical to matrix multiplication except that the scalars are convolved rather than multiplied. The convolutions are two-dimensional, because some of the matrices by nature are functions of both range, $r$, and azimuth, $x$, and 
in order to unify the notation all matrices are expressed as twodimensional quantities. For instance the TWT output is expressed by

$$
\mathbf{E}_{r}=\left[\begin{array}{cc}
e_{r}(r) \delta(x) & 0 \\
0 & e_{r}(r) \delta(x)
\end{array}\right]=e_{r}(r) \delta(x) \mathbf{I}
$$

where $e_{r}$ is the range encoding, and $I$ is the identity matrix.

Similar expressions can be set up for the other matrices, using Fig. 1 (Dall, 1994). Unlike $\mathbf{E}_{\mathbf{r}}, \mathbf{T}, \mathbf{E}_{\mathrm{a}}$ and $\mathbf{R}$, the matrices $\mathbf{C}_{\mathbf{S}}, \mathbf{G}$, and $\mathbf{A}_{\text {eff }}$ are not diagonal matrices because the polarisation switch and the antenna have finite channel separations and hence introduce crosstalk.

The measured SAR data are focused with range and azimuth filters $\left(\boldsymbol{H}_{\mathrm{r}}, \mathbf{H}_{\mathrm{a}}\right)$, and by changing the sequence of the matrices, the processed signal, $\mathbf{P}$, can be expressed in terms of a two-dimensional PTR, $\mathbf{H}_{2 \mathrm{D}}$

$$
\begin{aligned}
\mathbf{P} & =\mathbf{H}_{a} * \mathbf{H}_{r} * \mathbf{M}=\left(\mathbf{H}_{a} * \mathbf{E}_{a}\right) *\left(\mathbf{H}_{r} * \mathbf{R} * \mathbf{T} * \mathbf{E}_{r}\right) * \mathbf{A} * \mathbf{S} * \mathbf{G} * \mathbf{C}_{S} \\
& =\mathbf{H}_{2 D} * \mathbf{A} * \mathbf{S} * \mathbf{G} * \mathbf{C}_{S}=\mathbf{H}_{2 D} \tilde{\mathbf{A}} * \mathbf{S} \tilde{\mathbf{G}} \tilde{\mathbf{C}}_{S}
\end{aligned}
$$

The last equation utilises the fact that since $A, G$ and $C_{S}$ are assumed proportional to two-dimensional $\delta$-functions, the matrix convolutions degenerate into multiplications by matrices from which the $\delta$-functions have been removed (indicated with $\sim$ ).

$\mathbf{H}_{2 \mathrm{D}}$ is a diagonal matrix, but due to $\mathbf{R}$ the $\mathrm{H}$ and $\mathrm{V}$ channel IPRs are not identical

$$
\mathbf{H}_{2 D}=\frac{C_{m 4} C_{A} h_{a}(x)}{\sqrt{4 \pi} R^{2}}\left[\begin{array}{cc}
C_{P, H} h_{r, H}(r) & 0 \\
0 & C_{P, V} h_{r, V}(r)
\end{array}\right]
$$

\section{CALIBRATION}

A point target with radar cross section $\sigma_{i j}$ at polarisation $\mathrm{i}, \mathrm{j}$ has the scattering matrix $S_{i j}=\delta(r, x) \sqrt{\sigma_{i j} /(4 \pi)} e^{j \varphi_{i j}}$, where the phase depends on both the scattering mechanism and the propagation phase. The purpose of the calibration is to provide the image

$\mathbf{P}_{c a l}=\hat{\mathbf{H}}_{2 D}^{-1} \mathbf{H}_{2 D} * \mathbf{S}$

because in this image the above point target has the desired calibration integral

$$
\hat{\mathbf{P}}_{c a l}=\hat{\mathbf{S}}=\frac{1}{\sqrt{4 \pi}}\left[\begin{array}{ll}
\sqrt{\sigma_{H H}} e^{j \varphi_{H I I}} & \sqrt{\sigma_{H V}} e^{j \varphi_{H V}} \\
\sqrt{\sigma_{V H}} e^{j \varphi_{V H}} & \sqrt{\sigma_{V V}} e^{j \varphi_{V V}}
\end{array}\right]
$$

It is emphasized that the calibrated scene, $\mathbf{P}_{\text {cal }}$, is not identical to the original scattering matrix, $\mathbf{S}$, just because the point target has the same calibration integral. $\mathbf{P}_{\mathrm{cal}}$ is still a filtered version of $\mathbf{S}$.

The calibration result in Eq. 4 is achieved by a series of matrix pre- and post-multiplications which basically eliminate the matrices surrounding $\mathbf{S}$ in Eq. 3. The matrices are removed from outside like an onion is pealed

$$
\begin{aligned}
\mathbf{P}_{c a l} & =\left(\tilde{\mathbf{A}}^{-1} \hat{\mathbf{H}}_{2 D}^{-1}\right) \mathbf{P}\left(\tilde{\mathbf{C}}_{S}^{-1} \tilde{\mathbf{G}}^{-1}\right) \\
& =\left(\tilde{\mathbf{A}}^{-1}\left(\hat{\mathbf{H}}_{2 D}^{-1} \mathbf{H}_{2 D}\right) \tilde{\mathbf{A}}\right) * \mathrm{~S}\left(\tilde{\mathbf{G}}\left(\tilde{\mathbf{C}}_{S} \tilde{\mathbf{C}}_{S}^{-1}\right) \tilde{\mathbf{G}}^{-1}\right)
\end{aligned}
$$

As the antenna cannot be included in any internal calibration, the gain and directivity of the EMISAR antenna have been measured in the TUD-ESA spherical near-field test facility. The measurement itself is very accurate, so the $\tilde{\mathbf{A}}^{-1}$ and $\tilde{\mathbf{G}}^{-1}$ uncertainties are primarily determined by the steering of the three-axis stabilised antenna and the aircraft attitude measurement.
If the $\mathrm{H}$ and $\mathrm{V}$ channel IPRs are proportional, then

$\hat{\mathbf{H}}_{2 D}^{-1} \mathbf{H}_{2 D}=\left[\begin{array}{cc}\frac{h_{r, H}(r)}{\hat{h}_{r, H}} \frac{h_{a}(x)}{\hat{h}_{a}} & 0 \\ 0 & \frac{h_{r, v}(r)}{\hat{h}_{r, V}} \frac{h_{a}(x)}{\hat{h}_{a}}\end{array}\right]=\left[\begin{array}{cc}h_{H}(r, x) & 0 \\ 0 & h_{v}(r, x)\end{array}\right]$

is proportional to the identity matrix and hence it can be interchanged with $\tilde{\mathbf{A}}$, thereby reducing $\mathbf{E q}$. 7 to the desired result in Eq. 5. This issue is further discussed in the section on cross-talk elimination.

The internal calibration allows an in-flight determination of $\mathbf{H}_{2 D}$ and hence $\mathbf{H}_{2 D}^{-1}$. The normal calibration signal, $\mathbf{P}_{\mathbf{n}}$ (bold $\mathrm{TX}$ switch, shaded RX switches in Fig. 1) basically calibrates the TWT and the receivers, while the ratio of the two extended calibration signals covers the preamplifiers, and several waveguide and coax connections. The extended outer loop signal, $\boldsymbol{P}_{\mathrm{eol}}$, is acquired with the TX switch in the shaded position, the RX switches in the bold positions and the shutter switches closed. The extended inner loop signal, $\mathbf{P}_{\text {eij, }}$, is acquired with the TX and RX switches in the shaded positions.

Like the backscattered signal in Eq. $3, \mathbf{P}_{\mathrm{n}}, \mathbf{P}_{\text {eol }}$, and $\mathbf{P}_{\text {eil }}$, can be expressed as matrix convolutions, and when the components and subsystems in Fig. I are inserted and the calibration integrals computed, Dall (1994) has shown that

$\hat{\mathbf{H}}_{2 D}=\frac{-\hat{h}_{a}(x)}{\sqrt{4 \pi} R^{2}} \frac{C_{C, e}}{C_{C, n}} \frac{C_{m 3}}{C_{m 1}} \frac{C_{m 4}}{C_{m 2}}\left[\begin{array}{cc}\frac{\hat{P}_{c o l, H H}}{\hat{P}_{e i l, H H}} \frac{\hat{P}_{n, H H}}{C_{S, H H}} & 0 \\ 0 & \frac{\hat{P}_{e o l, V V}}{\hat{P}_{e i l, V V}} \frac{\hat{P}_{n, V V}}{C_{S, V V}}\end{array}\right]$

Here $h_{a}$ is the azimuth IPR which is well-known because it is primarily determined by a digital filter. $\mathrm{R}$ is the slant range which is measured very accurately by the SAR itself. $C_{C, e}$ and $C_{C_{n},}$ characterise the calibration attenuator in the extended and normal calibration modes, respectively, and since the calibration attenuator is a digitally controlled component with a high precision, the ratio is well-known. The only parameters to be measured in the laboratory are those of the directional coupler and the polarisation switch. The directional coupler is a simple passive component located in a temperature stabilised environment, so the laboratory measurements are reliable. The polarisation switch may be more critical as discussed in the section on the results. However, due to the $\tilde{\mathbf{C}}_{S}^{-1}$ matrix in Eq. 7 the polarisation switch cancels from the calibration of the co-polarised channels, and the cross-polarised channels depend only the ratio of the $\mathrm{HH}$ and VV parameters.

In $\mathrm{Eq} .9$ the calibration integrals computed from the normal calibration signals have cancelled the range IPR thereby calibrating the transmitter and receiver, and the ratios of the extended calibration integrals have cancelled the pre-amplifiers with adjacent connections, cf. Eq. 4.

\section{CROSS-TALK ELIMINATION}

The prime cross-talk contributors are the transmitting antenna, $\tilde{\mathbf{G}}$ and the receiving antenna $\tilde{\mathbf{A}}$. In the EMISAR case the cross-talk of the polarisation switch, $\overline{\mathbf{C}}_{S}$, can be neglected because it is about $12 \mathrm{~dB}$ lower than that of the antennas.

The correction for the antennas is conveniently split into a channel imbalance correction and a cross-talk elimination 


$$
\begin{aligned}
\tilde{\mathbf{G}}^{-1} & =\frac{1}{G_{H H} G_{V V}-G_{H V} G_{V H}}\left[\begin{array}{cc}
G_{V V} & -G_{H V} \\
-G_{V H} & G_{H H}
\end{array}\right]=\left[\begin{array}{cc}
\frac{1}{G_{H H}} & \frac{-G_{H V}}{G_{H H} G_{V V}} \\
\frac{-G_{V H}}{G_{H H} G_{V V}} & \frac{1}{G_{V V}}
\end{array}\right] \\
& =\left[\begin{array}{cc}
\frac{1}{G_{H H}} & 0 \\
0 & \frac{1}{G_{V V}}
\end{array}\right]\left[\begin{array}{cc}
1 & \frac{-G_{H V}}{G_{V V}} \\
\frac{-G_{V H}}{G_{H H}} & 1
\end{array}\right] \approx \tilde{\mathbf{G}}_{D}^{-1} \tilde{\mathbf{G}}_{x}^{-1} \\
\tilde{\mathbf{A}}^{-1} & =\left(\frac{\lambda}{\sqrt{4 \pi}} \tilde{\mathbf{G}}^{T}\right)^{-1} \\
& =\left[\begin{array}{cc}
1 & \frac{-G_{V H}}{G_{H H}} \\
\frac{-G_{H V}}{G_{V V}} & 1
\end{array}\right]\left[\begin{array}{cc}
\frac{\sqrt{4 \pi}}{\lambda G_{H H}} & 0 \\
0 & \frac{\sqrt{4 \pi}}{\lambda G_{V V}}
\end{array}\right] \approx \tilde{\mathbf{A}}_{x}^{-1} \tilde{\mathbf{A}}_{D}^{-1}
\end{aligned}
$$

Second order cross-talk terms are neglected.

The matrices are decomposed so that $\tilde{\mathbf{A}}_{D}^{-1}$ and $\tilde{\mathbf{G}}_{D}^{-1}$ become the innermost matrices, with the implication that the channel imbalance corrections are performed first. These corrections must be implemented in the range-Doppler domain, as the antenna patterns are functions of both the elevation angle, $\varphi_{\mathrm{e}}$ and the azimuth angle, $\varphi_{\mathrm{x}}$. When the SAR processing is based on the range-Doppler algorithm, the corrections are conveniently combined with the azimuth focusing.

Eqs. 10 and 11 show that unless $G_{V H}\left(\varphi_{x}\right)$ and $G_{H H}\left(\varphi_{x}\right)$ are proportional, and similarly $\mathrm{G}_{\mathrm{HV}}\left(\varphi_{\mathrm{x}}\right)$ and $\mathrm{Gvv}_{\mathrm{V}}\left(\varphi_{\mathrm{x}}\right)$, the cross-talk elimination, too, depend on the azimuth angle. This is important because in this case the cross-talk can be only partly eliminated in the time domain, where the correction is usually implemented. Fig. 2 illus trates that the co- and cross-polarised patterns are not necessarily proportional.

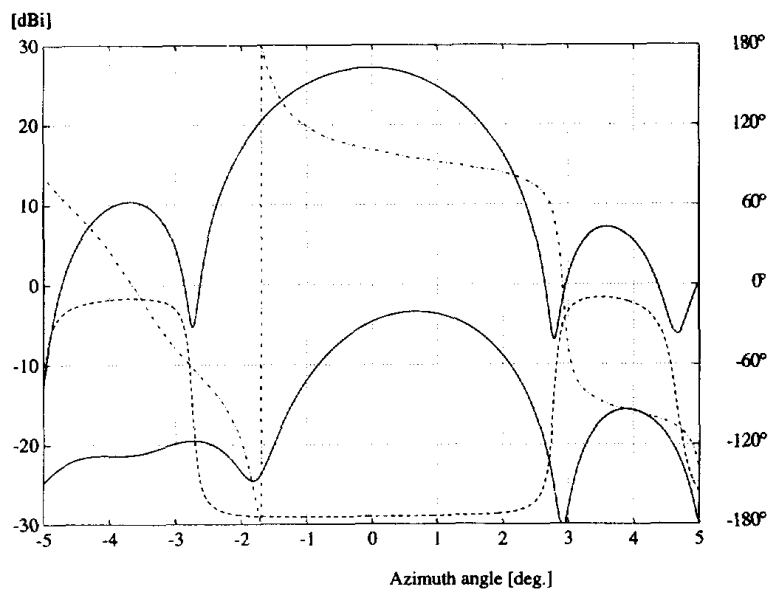

Fig. 2. EMISAR HH and VH azimuth patterns (amplitudes: solid lines, HH phase: dashed line, VH phase: dash-dot line).

What the multiplications by $\tilde{\mathbf{A}}_{X}^{-1}$ and $\tilde{\mathbf{G}}_{X}^{-1}$ actually do is to subtract the cross-talk signals. This can be illustrated by evaluating Eq. 7

$$
\begin{aligned}
\mathbf{P}_{c a l} & =\left(\tilde{\mathbf{A}}_{X}^{-1} \tilde{\mathbf{A}}_{D}^{-1}\left(\hat{\mathbf{H}}_{2 D}^{-1} \mathbf{H}_{2 D}\right) \tilde{\mathbf{A}}_{D} \tilde{\mathbf{A}}_{X}\right) * \mathrm{~S}\left(\tilde{\mathbf{G}}\left(\tilde{\mathbf{C}}_{S} \tilde{\mathbf{C}}_{S}^{-1}\right) \tilde{\mathbf{G}}^{-1}\right) \\
& =\tilde{\mathbf{A}}_{x}^{-1}\left(\hat{\mathbf{H}}_{2 D}^{-1} \mathbf{H}_{2 D}\right) \tilde{\mathbf{A}}_{X} * \mathbf{S}
\end{aligned}
$$

If $\hat{\mathbf{H}}_{2 D}^{-1} \mathbf{H}_{2 D}$ is neglected for a moment, we find

$\tilde{\mathbf{A}}_{X}^{-1} \overline{\mathbf{A}}_{X} * \mathbf{S}=\left[\begin{array}{cc}1 & \frac{-G_{V H}}{G_{H H}} \\ \frac{-G_{H V}}{G_{V V}} & 1\end{array}\right]\left[\begin{array}{ll}S_{H H}+\frac{G_{V H}}{G_{H H}} S_{V H} & s_{H V}+\frac{G_{V H}}{G_{H H}} S_{V V} \\ S_{V H}+\frac{G_{H V}}{G_{V V}} S_{H H} & S_{V V}+\frac{G_{H V}}{G_{V V}} S_{H V}\end{array}\right]$

where for instance the $\mathrm{HH}$ element is obtained by adding the $\mathrm{VH}$ element after a scaling by $-G_{\mathrm{VH}} / \mathrm{G}_{\mathrm{HH}}$. (The reason why the result is not exactly $S_{H H}$ is that second order cross-talk terms were neglected in Eqs. 10 and 11). If the scale factor is a function of the azimuth angle and hence the Doppler frequency, the scaling is actually a filtering that equalises the VH azimuth IPR to the HH cross-talk. The cross-talk is not completely eliminated unless the subtrahend has the same IPR.

It may also be necessary to equalise the range IPR. The impact of $\hat{\mathbf{H}}_{2 D}^{-1} \mathbf{H}_{2 D}$ is that the rows of the last matrix in Eq. 13 are multiplied by $h_{H}(r, x)$ and $h_{V}(r, x)$, respectively. The consequences are the same as for the azimuth IPR. An equalisation can be based on the internal calibration signals (Dall, 1994).

The range IPR does not impact the multiplication by $\tilde{\mathbf{G}}_{X}^{-1}$ because channels in different columns are combined, not rows. However, with respect to the azimuth IPR the above comments do apply, and moreover, since channels with different transmit polarisation are combined, these channels must register. Therefore, the azimuth resampling that corrects for the fact that the $\mathrm{H}$ and $\mathrm{V}$ polarised pulses are transmitted alternately, must precede the multiplication by $\tilde{\mathbf{G}}_{X}^{-1}$.

\section{RESULTS}

The impact of the EMISAR range and azimuth IPRs has been examined by simulations. When the calibration integrals $\hat{P}_{n, H H}$ and $\hat{P}_{n, W}$ in Eq. 9 are replaced by the amplitude and phase of a calibration tone close to the upper frequency of the transmitted spectrum, a calibration error of about $1.5 \mathrm{~dB}$ is introduced. This error depends strongly on the pulse coding, the relative bandwidth and the weighting used in the SAR processor for sidelobe reduction.

An ideal cross-talk elimination (complete suppression) has been compared to one implemented in the time domain instead of the range-Doppler domain. The latter was able to suppress the cross-talk by $22 \mathrm{~dB}$. Also, the ideal cross-talk elimination has been compared to one with no range equalisation. The latter was able to suppress the cross-talk by $17 \mathrm{~dB}$. Since EMISAR's cross-talk before correction is about -30 dB (Skriver, 1994), it can be concluded that range-Doppler domain implementation and range equalisation are not mandatory.

The full set of pre- and post-map calibration pulses were acquired during a calibration experiment conducted at DLR, Oberphaffenhofen near München, Germany, prior to the EMAC campaigns (European Multisensor Airborne Campaign) arranged by the European Space Agency (ESA) in co-operation with the Joint Research Centre (JRC).

The DLR test site with a total of 9 trihedrals and 4 dihedrals was mapped several times, and the data were processed and calibrated according to the procedure presented in this paper with the exception that the antenna cross-talk matrices, $\overline{\mathbf{A}}_{X}^{-1}$ and $\overline{\mathbf{G}}_{X}^{-1}$ in Eqs. 10 and 11 , were excluded (they did not reduce the cross-talk significantly). By means of external calibration the residual calibration errors in Table 1 were found (average \pm standard deviation based on 9 estimates). The combination of trihedrals and dihedrals with an orientation angle of $45^{\circ}$ allowed the HH-VV channel imbalance to be decomposed into imbalances on the transmit and receive sides. This method has an intrinsic $180^{\circ}$ phase ambiguity which was resolved with an additional $22.5^{\circ}$ dihedral. 
TABLE 1. Residual calibration errors.

\begin{tabular}{|l|c|c|}
\hline & Amplitude [dB] & Phase [deg] \\
\hline Abs. calibration & $-1.95 \pm 0.34$ & NA \\
\hline TX imbalance & $0.13 \pm 0.28$ & $11.0 \pm 2.9$ \\
\hline RX imbalance & $0.94 \pm 0.27$ & $133.1 \pm 3.5$ \\
\hline
\end{tabular}

The residual calibration crrors are larger than expected. Potential errors include: bugs in the data calibration software, erroneous component measurements, crroncous model assumptions, lack of system stability. So far, the reason has not been found.

The system has been subject to extensive measurements, and more are planned, e.g. the impact of the low pod temperature on the antenna will be examined.

To some extent the system stability can be assessed by means of the internal calibration pulses. The normal calibration pulses and the extended inner loop pulses have proved reasonable stable, i.e. drift less than $0.04 \mathrm{~dB}$ and $3^{\circ}$ from a pre-map calibration to the corresponding post-map calibration and less than $0.3 \mathrm{~dB}, 3^{\circ}$ from one map to another within the same day. From these observations it can be concluded that the temperature stabilised equipment in the aircraft cabin, and in particular the TWT, does not cause much drift.

The amplitude drift of the extended outer loop pulses is less than $0.17 \mathrm{~dB}$ from pre-map to post-map and from one map to another. Relative to the $\mathrm{HH}$ phase the VV phase drifts less than $2^{\circ}$ from pre-map to post-map and from one map to another. It is interesting that the absolute phase drifts about $30^{\circ}$ from pre-map to postmap, but less than $1^{\circ}$ from one pre-map to another pre-map and from one post-map to another post-map. This suggests that the absolute drift is caused by a heating within the polarisation switch taking place during mapping, which has been verified in the laboratory.

The cross-talk of the polarisation switch can be measured directly from the quad-polarised extended outer loop pulses, (Dall, 1994). It is suppressed by 36 to $47 \mathrm{~dB}$, but it varies up to $10 \mathrm{~dB}$ over the $100 \mathrm{MHz}$ bandwidth, and it drifts 4 to $7 \mathrm{~dB}$ during the mapping. Therefore, it is hardly possible to eliminate the cross-talk introduced by the polarisation switch.

\section{CONCLUSION}

The pulse-based internal calibration procedure has been shown to include almost the entire SAR system, and to take the point target response into account. A preliminary evaluation shows that the internal calibration and external calibration differ 1 to $2 \mathrm{~dB}$. These deviations are presently not well understood, and we consider them unacceptable. Currently we are looking into possible causes, e.g the performance of critical components such as the shutter switches.

\section{ACKNOWLEDGEMENT}

The authors thank A. Netterstrom, S.S. Kristensen and S.N Madsen who have contributed with control and processing software, laboratory measurements and many valuable comments. The work and data acquisition was funded by the Danish Technical Research Council, the Joint Research Centre, the European Space Agency, the Danish National Science Foundation, and the Technical University of Denmark.

\section{REFERENCES}

Christensen, E.L., S.N. Madsen, J. Dall, N. Skou, K. Woelders, A. Netterstrøm, J.H. Jørgensen, J. Granholm, M. Dich, "The Danish Polarimetric SAR for Remote Sensing Applications", in International Geoscience and Remote Sensing Symposium Digest, 1994.

Dall, J., "Internal Polarimetric Calibration", R576, Electromagnetics Institute, Tcchnical University of Denmark, Lyngby, Denmark, February, 1994, 56 pp.

Gray, A.L., P.W. Vachon, C.E. Livingstone and T.I. Lukowski, "Synthetic Aperture Radar Calibration Using Reference Reflectors", IEEE Transactions on Geoscience and Remote Sensing, Vol. 28, No. 3, May 1990, pp. 374-383.

Skou, N., "The Danish Polarimetric SAR System", Proceedings of Second International Workshop on Radar Polarimetry, Nantes, France, September, 1992, pp. 525-533.

Skriver, H., J. Dall, and S.N. Madsen, "External Polarimetric Calibration of the Danish Polarimetric C-band SAR", in International Geoscience and Remote Sensing Symposium Digest, 1994. 